\title{
ALTMAN TYPE 3 SACROCOCCYGEAL TERATOMA RESECTION IN A NEONATE- AN ANAESTHETIC CHALLENGE
}

\author{
Suchismita Mallick1, Sarbari Swaika2, Sarmila Ghosh³, Pijush Kanti Choudhury4
}

${ }^{1}$ Assistant Professor, Department of Anaesthesiology \& Critical Care, Institute of Post-Graduate Medical Education and Research \& SSKM Hospital, Kolkata.

${ }^{2}$ Associate Professor, Department of Anaesthesiology \& Critical Care, Institute of Post-Graduate Medical Education and Research \& SSKM Hospital, Kolkata.

${ }^{3}$ Associate Professor, Department of Anaesthesiology, Critical Care \& Pain Medicine, RG Kar Medical College, Kolkata.

${ }^{4}$ Senior Resident, Department of Anaesthesiology \& Critical Care, Institute of Post-Graduate Medical Education and Research \& SSKM Hospital, Kolkata.

\section{ABSTRACT}

\section{BACKGROUND}

Sacrococcygeal teratoma (SCT) is the commonest tumour in neonates occurring in 1: 40,000 births. Early removal of the tumour is advised in neonates as there is increased chance of malignant transformation with age. There are several reports on the haemorrhage associated with the tumour, complications of massive blood transfusion and even cardiac arrest in neonates. Here, we present successful anaesthetic management in a similar case of type 3 sacrococcygeal tumour resection in a $3.6 \mathrm{~kg}$ full term female child with vascular injury resulting in massive haemorrhage in operation theatre. Despite intractable haemorrhage, the child was saved with momentary clamping of internal iliac artery along with adequate intravenous fluid and blood transfusion. Child was on ventilatory support for 5 days postoperatively and finally extubated when she was maintaining well with oxygen saturation of $97 \%$ in room air. SCT resection is associated with problems like massive haemorrhage, coagulopathy, hypothermia, hyperkalaemia, ventilatory difficulties and features of hydrops that is due to high output cardiac failure. Apart from the considerations of neonatal anaesthesia, there should be anticipation of these problems and preparation of emergency medications and requisition of blood and blood products need to be done.

\section{KEYWORDS}

Sacrococcygeal Teratoma, Massive Haemorrhage, Blood Transfusion.

HOW TO CITE THIS ARTICLE: Mallick S, Swaika S, Ghosh S, et al. Altman type 3 sacrococcygeal teratoma resection in a neonateAn anaesthetic challenge. J. Evolution Med. Dent. Sci. 2017;6(17):1388-1390, DOI: 10.14260/Jemds/2017/303

\section{BACKGROUND}

Sacrococcygeal teratoma (SCT) is the commonest tumour in neonates occurring in 1: 40,000 births. Early removal of the tumour is advised in neonates as there is increased chance of malignant transformation with age. There are several reports on the haemorrhage associated with the tumour, complications of massive blood transfusion and even cardiac arrests in neonates. ${ }^{1}$ Here, we report a similar case of type 3 sacrococcygeal tumour resection with vascular injury resulting in massive haemorrhage managed successfully in operation theatre (OT).

\section{CASE REPORT}

A $3.6 \mathrm{~kg}$ full term female baby born by emergency caesarean section presented to the neonatology OPD on $26^{\text {th }}$ day with a chief complaint of a sacral mass. It was a cystic swelling at sacral region gradually increasing in size. On examination, an intra-abdominal mass $6-8 \mathrm{~cm}$ was palpated which was cystic to firm at places. Other systemic examinations were within normal limits except swelling and oedema of lower extremities extending upwards.

Financial or Other, Competing Interest: None.

Submission 28-12-2016, Peer Review 14-02-2017,

Acceptance 20-02-2017, Published 27-02-2017.

Corresponding Author:

Dr. Sarbari Swaika

Associate Professor,

Department of Anaesthesiology \& Critical Care,

Institute of Post Graduate Medical Education and

Research \& SSKM Hospital,

Kolkata.

E-mail:dr.s.swaika@gmail.com

DOI: $10.14260 /$ jemds $/ 2017 / 303$

\section{(c) (i) $(-)$}

Pre-operative investigations were within normal limits. MRI revealed a thick-walled cystic space occupying lesion noted in the sacral region with intrapelvic components. It was echogenic with foci of calcification and was diagnosed as type 3 sacrococcygeal teratoma.

Patient was posted for exploratory laparotomy for excision of intra-abdominal mass. On arrival to OT, monitors like noninvasive blood pressure monitoring (NIBP), electrocardiogram, oxygen saturation, capnography and temperature monitoring were attached.

Preoxygenation with $100 \%$ oxygen was done for 3 minutes; premedication with Inj. Atropine $80 \mu \mathrm{g}$ and Inj. Fentanyl $8 \mu \mathrm{g}$ was given. Patient was induced with sevoflurane inhalation; muscle relaxant Inj. Atracurium was used. Intravenous paracetamol $75 \mathrm{mg}$ was given. Intraoperative maintenance of anaesthesia was done with $0.4 \%$ isoflurane and oxygen. When the tumour vessels were being ligated there was a sudden gush of bleeding. A vascular injury was detected and internal iliac artery was momentarily clamped to control the intractable haemorrhage. Peripheral pulses in femoral artery were palpable and skin colour was identical in both the lower limbs and were repeatedly checked to rule out loss of vascular supply to the lower limbs. NIBP was maintained in the intra-operative period with adequate intravascular fluid therapy and blood transfusion. A total of $270 \mathrm{~mL}$ ringer lactate and $180 \mathrm{~mL}$ whole blood was given. A small bolus of $10 \mathrm{mg}$ calcium gluconate (10\%)- 0.5 $\mathrm{mL}$ diluted in $1 \mathrm{~mL}$ of water was given. There was not a single episode of hypotension or fall in oxygen saturation during the entire intra-operative period. The tumour was excised and rest of the intra-operative period was uneventful. Patient was 
sent to NICU for elective ventilation and further management. Urine output at the end of operation was $60 \mathrm{~mL}$.

Immediate postoperative investigations revealed $\mathrm{Hb}-6.2$ $\mathrm{g} / \mathrm{dL}$, Hct-25\%, and total WBC count-4800. Serum electrolytes were Na-133.8, K-4.01, Ca-1.126, Cl-99.5. Patient was put on a ventilator setting of SIMV with PSV. FiO2-80\%, PEEP-5, Minute ventilation-1.3 L/min., Respiratory rate40/min., Tidal volume of $33 \mathrm{~mL}$, I:E-1:2. Patient was maintaining a saturation of $80-90 \%$. Inj. Dopamine was started @10 $\mu \mathrm{g} / \mathrm{kg} / \mathrm{min}$. Inj. Vit. K and tranexamic acid was given. Patient received $65 \mathrm{~mL}$ of packed RBC and $60 \mathrm{~mL}$ of FFP in the immediate postoperative period.

Patient was on ventilator support for the next 5 days with gradual tapering of the settings and weaning off. Patient was finally extubated and was maintaining well with oxygen saturation of $97 \%$ in room air. Feeding was started the next morning with breast milk and the patient was discharged later.

\section{DISCUSSION}

There are 4 types of sacrococcygeal teratomas. The neonate in this case was diagnosed with Altman type 3 SCT which has both an intra-abdominal presacral and extra-abdominal presentation. ${ }^{2,3}$ This tumour has a female preponderance and factors like antepartal diagnosis before 20 weeks of gestation, prematurity, LBW, low APGAR scores, placentomegaly, nonimmune hydrops, polyhydramnios and malignant histotypes have worse prognosis. ${ }^{4}$ In this case, it was a full term male baby with normal APGAR score with a postpartal diagnosis of SCT. The baby had evidence of hydrops with generalised oedema but no respiratory difficulty. Hydrops is due to arteriovenous shunting from a large vascular tumour leading to high output cardiac failure. It carries poor prognosis and is associated with high mortality. ${ }^{5}$ It sometimes results in a mirror syndrome where the foetal hydrops causes placentomegaly and ultimately features of maternal hydrops with preeclampsia and oedema develop which requires urgent delivery of the foetus. ${ }^{6}$

Complete excision of the SCT including coccygectomy is advised to avoid recurrence.7,8 Hypervascularity of the tumour has been described in previous reports, the arterial supply being middle sacral artery which is a branch of internal iliac artery. Apart from the high vascularity, associated coagulopathy which becomes worse with time and hypothermia are contributory factors to haemorrhage. In this case, the bleeding was managed initially with packing and surgically ligating the internal iliac artery which is the source of the main feeder. During the haemorrhagic episode, blood transfusion in aliquots of $20-30 \mathrm{~mL}$ of whole blood was done along with liberal fluid resuscitation with RL. Total volume of $180 \mathrm{~mL}$ of whole blood was given in a short span of time and with the ongoing bleeding, a small bolus of $10 \mathrm{mg}$ calcium gluconate $(10 \%)-0.5 \mathrm{~mL}$ diluted in $1 \mathrm{~mL}$ of water was given to counter the effects of hypocalcaemia and hyperkalaemia associated with the blood transfusion. In the other cases, various amounts of PRBC, FFP and platelets were given along with crystalloids. Blood loss is difficult to assess as there might be hidden loss inside the necrotic tissues in the tumour. In one study, around $300 \mathrm{~mL}$ of blood loss was replaced with PRBC $320 \mathrm{~mL}$, FFP $43 \mathrm{~mL}$, platelets $40 \mathrm{~mL}$, cryoprecipitate $20 \mathrm{~mL}$, and crystalloid $90 \mathrm{~mL} .1$ Transfusion in neonates and infants less than 4 months' age is frequently needed and different from older children as this population has a smaller blood volume and does not tolerate blood loss well. Target haematocrit is $35 \%$ for neonates with severe respiratory distress. The erythropoietic response is not developed and the cardiac output is maintained by increase in heart rate only. The incidence of massive blood transfusion is more in this population and complications such as acidosis, alkalosis (citrate gets converted to bicarbonate in the liver), hypocalcaemia, hyperglycaemia (from the stored glucose in PRBC), hyperkalaemia and hypothermia are common. Apart from trauma victims where early use of FFP is advised, FFP is usually needed when about 1.5 times the blood volume is lost and platelets are required when about 2 times the blood volume is lost. The coagulopathy that occurs after massive blood transfusion in neonates is mainly due to coagulation factor dilution and FFP is needed to correct it. In acute settings PT takes around 45-60 minutes to obtain, so TEG is emerging as a method for coagulation status assessment in recent times. In planned OT blood products need to be kept in hand according to the case and in anticipation and repeated blood transfusion should ideally be given from the same unit to avoid multiple donors. After massive transfusion, blood should be cross matched and given in consultation with the haematologist.

Another problem in these cases is electrolyte imbalance particularly hyperkalaemia which can be due to tumour handling in intra-abdominal tumours, respiratory and metabolic acidosis, hypocalcaemia and also from massive PRBC transfusion.9,10,11 It can be managed with NaHCO3 1 $\mathrm{mEq} / \mathrm{kg}$ slow IV, Ca gluconate- $2 \mathrm{~mL}$ double diluted and given slowly, insulin in dextrose.

Hypothermia is also common due to exposure of the large surface area of the tumour, rapid administration of fluids and should be aggressively managed with warmed fluids and active warming methods.

Abraham described a case of ventilatory difficulty from an intra-abdominal tumour pressing on the diaphragm, managed with lifting of the tumour. This was observed in case of intraabdominal resection in supine position and resulted in increase in peak airway pressure $>25 \mathrm{~mm} \mathrm{H}_{2} \mathrm{O}$, reduced tidal volume and ABG changes and also cardiac arrest. ${ }^{4}$ No such problem was encountered in this case.

\section{CONCLUSION}

To conclude, SCT resection is associated with problems like massive haemorrhage, coagulopathy, hypothermia, hyperkalaemia, ventilator difficulties and features of hydrops that is due to high output cardiac failure. Apart from the considerations of neonatal anaesthesia, there should be anticipation of these problems and preparation of emergency medications and requisition of blood and blood products need to be done. With proper preparations, even the most challenging scenarios can be tackled. One question arising from this case report is the utilisation of hypotensive anaesthesia for identification of the bleeder in case of acute bleeding and the relative volumes of resuscitative fluids used and their impact on the outcome of the case.

\section{REFERENCES}

[1] Girwalkar-Bagle A, Thatte WS, Gulia P. Sacrococcygeal teratoma: a case report and review of literature. Anaesth Pain \& Intensive Care 2014;18(4):449-51. 
[2] Isaacs H. Perinatal (fetal and neonatal) germ cell tumors. J Pediatr Surg 2004;39(7):1003-13.

[3] Ashley DJ. Origin of teratomas. Cancer 1973;32(2): 390-4.

[4] Abraham E, Parray T, Ghafoor A. Complications with massive sacrococcygeal tumor resection on a premature neonate. J Anesth 2010;24(6):951-4.

[5] Robertson FM, Crombleholme TM, Frantz ID, et al. Devascularization and staged resection of giant sacrococcygeal teratoma in the premature infant. J Pediatr Surg 1995;30(2):309-11.

[6] Finamore PS, Kontopoulos E, Price M, et al. Mirror syndrome associated with sacrococcygeal teratoma: case report. J Reprod Med 2007;52(3):225-7.

[7] Legbo JN, Opara WEK, Legbo JK. Mature sacrococcygeal teratoma: case report. African Health Sci 2008;8(1): 54-7.
[8] Altman RP, Randolph JG, Lilly JR. Sacrococcygeal teratoma: American academy of pediatrics surgical section survey-1973. J Pediatr Surg 1974;9(3):389-98.

[9] Jung-Won K, Mijeung G, Jong-Yeon P, et al. Cardiac arrest during excision of a huge sacrococcygeal teratoma - a report of two cases. Korean J Anesthesiol 2012;63(1):80-4.

[10] Jona JZ. Progressive tumor necrosis and lethal hyperkalemia in a neonate with sacrococcygeal teratoma (SCT). J Perinatol 1999;19(7):538-40.

[11] Reinoso-Barbero F, Sepulveda I, Perez-Ferrer A, et al. Cardiac arrest secondary to hyperkalemia during surgery for a neonatal giant sacrococcygeal teratoma. Paediatr Anaesth 2009;19(7):712-4. 